\title{
Wormhole Network Imaging in Post-Chops Process Using Ground-Penetrating Radar
}

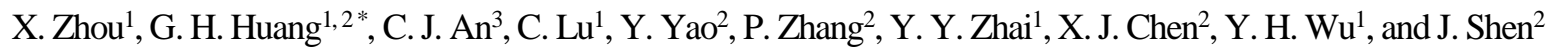 \\ ${ }^{1}$ Institute for Energy, Environment and Sustainable Communities, 240, 2 Research Drive, Regina, Saskatchewan, S4S 7H9 Canada \\ ${ }^{2}$ Department of Environmental Systems Engineering, University of Regina, 3737 Wascana Parkway, Regina, Saskatchewan, S4S 0A2 Canada \\ ${ }^{3}$ Department of Building, Civil and Environmental Engineering, Concordia University, Montreal, Quebec, H3G 1 M8 Canada
}

Received 13 March 2020; revised 1 May 2020; accepted 26 May 2020; published online 20 June 2020

\begin{abstract}
In this study, an integrated near-field GPR system (INFGPRs) with a frequency tunable patch antenna was developed to investigate wormholes in oil sand that emerge during CHOPS. 3-dimension imaging of wormholes was then obtained based on INFGPRs through step-scanning against the wall of inspection wells. The feasibility and availability of the developed GPR system were identified through bench-scale simulation in the lab. In addition, a factorial factor experiment was designed in order to reveal the interaction effects of impact factors (depth, size, and content of wormhole) on the performance of the developed GPR system.
\end{abstract}

Keywords: CHOPS, wormholes, factorial analysis

\section{Introduction}

Canada is the fourth-largest oil producer around the globe, where nighty-seven percent of its proven oil reserves are detected in oil sand (Chen et al., 2019a; Natural Resources Canada, 2019). Novel extraction technologies to maximize output are expected to be developed and applied in oil and gas industries, such as hydraulic fracturing, horizontal drilling, and bitumen extraction (Akhter and Azam, 2019). However, recovering heavy crude oil from an oil-bearing formation is known to be difficult. Cold heavy oil production with sand (CHOPS) is an effective extraction approach in which oil is extracted together with sand (Duisterwinkel et al., 2016). During the CHOPS process, channels are formed in the reservoir. These channels are known as wormholes (Liu and Zhao, 2004). A wormhole may penetrate several feet into a formation. Although wormholes allow the oil to flow, they may form a connection between two wells, causing water encroachment or pressure loss, which leads to the reduction of oil extraction.

In order to place CHOPS wells optimally, the spatial distribution of wormholes has been studied through experimental investigation and numerical simulation. High conductivity of wormholes was demonstrated by tracer tests, pressure tests, injection tests. (Duisterwinkel et al., 2016). A transient-pressure analysis was developed to detect the growth of wormholes in pilot CHOPS wells (Sanyal and Al-Sammak, 2011). In addition, a series of numerical-simulation tools were used to estimate wormhole footprints by historical matching field data to

* Corresponding author. Tel: +1 3065854095; fax: +1 3063373205. E-mail address: huang@iseis.org (G. H. Huang).

ISSN: 2663-6859 print/2663-6867 online

() 2020 ISEIS All rights reserved. doi:10.3808/jeil.202000030. analyze the effects of wormholes with enhanced permeability on increased production (Sawatzky et al., 2002). The effects of different wormhole patterns on transient-pressure behaviors were analyzed by source-and-sink-function methods which were expected to reproduce wormhole configurations (Liu and Zhao, 2005). Furthermore, a diffusion-limited aggregation algorithm was developed to identify wormhole patterns (Rangriz Shokri and Babadagli, 2014). Additionally, a dynamic wellbore module was implemented to model wormhole growth with multilateral wells (Istchenko and Gates, 2014).

Previous studies cannot provide visual status of wormholes. Ground-penetrating radar (GPR) is a high-resolution and non-destructive detection technology for subsurface investigation, imavgination, and diagnosis (Bell et al., 2005). The two-way travel time can be transformed to distance directly through the application of standard values of electromagnetic wave propagation rates for a variety of materials (Bell et al., 2005). Compared to seismic, transient electromagnetic, or electrical and magnetic approaches, GPR can provide denser data with higher accuracy. This innovative technology has been applied to the investigation of underground utilities (Saarenketo and Scullion, 2000), roots (Bain et al., 2017), voids (SHRP2, 2013), and water content (Khristoforov and Omelyanenko, 2018) successfully. GPR performance can be enhanced form the following three aspects: GPR frequency, antenna design, and data analysis. The antenna is a critical hardware component that has a direct influence on the range and resolution of a GPR system. The selection of the operational frequency is associated with a tradeoff between resolution and detection range: highfrequency radio wave can result in high-resolution imaging but penetrates a shallow depth, while low-frequency radio wave penetrates deep into the ground at the cost of image resolution. Hence, low-frequency antennas are more applicable to larger 
penetration depths, such as geological surveys, stratigraphic investigations, underground pipe, as well as for river/landfill and lake-bottom analysis (Pajewski et al., 2013). The performance of GPR can also be improved by having an ultra-wide frequency band and linear phase characteristics, as well as the coupling between transmitting and receiving antennas (Benedetto and Paiewski, 2015). The most frequently used GPR antennas are dipole, end-fire tapered slot, spiral, and horn antennas. Among these antenna configurations, the transverse electromagnetic horn antenna has been wildely employed in GPR systems due to its excellent time-domain characteristics (Gentili and Spagnolini, 2000). However, there are few reports of the GPR application on the imaging of wormholes in CHOPS.

The characteristics of oil sand present challenges for the GPR system. The depth of the wormholes is below 200 meters (Liu and Zhao, 2004). Detectable depth of GPR is related to GPR frequency and relative dielectric constant of the medium. The oil sand contains high salinity solutions that can significantly decrease the detectable range of GPR. Recently, lowfrequency submersible GPR systems (range from $15 \sim 75$ $\mathrm{MHz}$ ) were used to investigate water bodies with natural dissolved-solids contents (Khristoforov and Omelyanenko, 2018).

In this study, an integrated near-field GPR system (INFGPRs) with a frequency tunable patch antenna was developed to investigate wormholes in oil sand that emerge during CHOPS. INFGPRs was expected to obtain 3-dimension imaging of wormholes through step-scanning against the wall of inspection wells. The inspection wells are expected to be without any metal sleeve to improve signal transmission. Herein, feasibility and availability of the developed GPR system were identified through bench-scale simulation in the lab. In addition, a factorial factor experiment was designed in order to reveal the interaction effects of impact factors (depth, size, and filler of wormhole) on the performance of the developed GPR system. Furthermore, a robust signal processing methodology was developed to enhance the signal to noise ratio and create 3-dimension imaging of the wormholes.

\section{Build/Development of Lab-Scale Wormhole Simulation System}

The wormhole simulation site was established in a lab with a temperature of $25^{\circ} \mathrm{C}$ to avoid environmental disturbance. The mainframe, which was design to direct and continuous sample at depth along with vertical intervals, was constructed with polyvinyl chloride plastic plate, which was stacked with sands characterized by different porosities and sizes to simulate geological properties of oil drilling sites, as shown in Figure 1. One of the side surfaces was determined as origin surface, indicating start-up ground surface in real-world, where the up-left corner was set as coordination origin of X (width), Y (height), $\mathrm{Z}$ (length) axis. Different sizes of plastic vials were buried in the sand phase with constant $\mathrm{X}$ and $\mathrm{Y}$ value to represent wormholes in oil recovery progress. The vertical distances between origin surface and buried point can be considered as the depth of wormhole in real-world imaging.

A TerraSIRch Subsurface Interface Radar (SIR) System
3000 with a 400-MHz antenna (manufactured by Geophysical Survey Systems, Inc. (GSSI), Salem, NH), was used to imaging wormhole in this experiment. The $400-\mathrm{MHz}$ antenna can be used to provide a balanced signal between resolution and depth of penetration. A total of 48 scans were collected per second by using the SIR-3000 control unit, corresponding to 50 scans per meter. The result signals from radar were analyzed through RADAN for Windows 5.0 (GSSI). With focuses on imaging of wormhole under different conditions, the comparisons of wormhole depths, hole sizes, and medium fillers were conducted to investigate the performance of field acquisition and the effects of different factors on wormhole presentation. Different medium fillers were investigated, including clean water, crude oil and oil-water mixer (50/50 v). In the comparison experiments, non-independent factors were set as control values. The control values of depth, hole sizes, and medium filler (crude oil) were determined based on real-world application parameters and experiment purpose for distinguishable results.

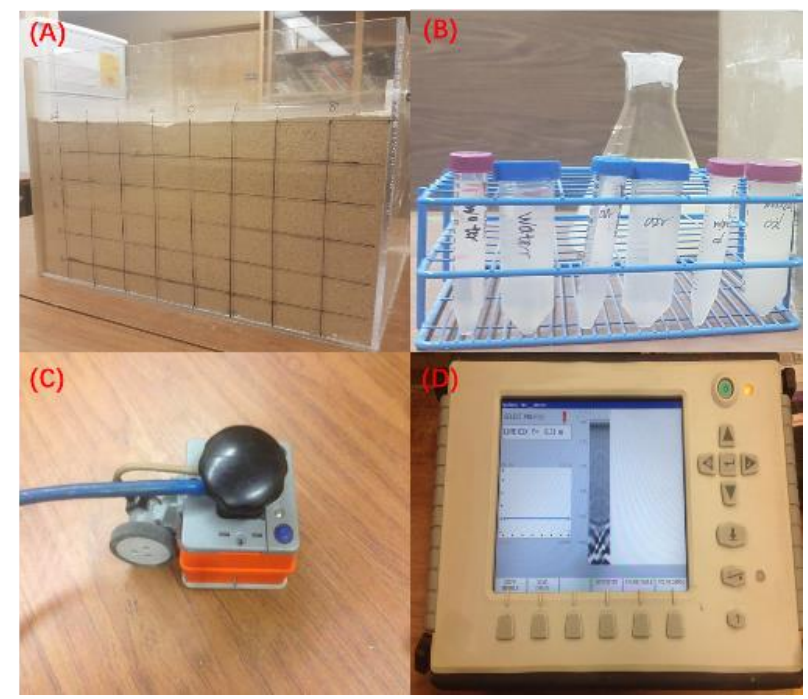

Figure 1. Pilot system for wormholes investigation by groundpenetrating radar (GPR). (A) BOX model of CHOPS site; (B) Models of wormholes; (C) Antenna of GPR; (D) Processer of GPR.

\section{Asymmetrical Factorial Design}

An asymmetrical factorial design is employed to reveal the interactions of wormhole depth, wormhole size and medium filler on imaging performance. The factorial design has been extensively performed to determine the main and interactive effects of factors in various studies (Zhou et al., 2016; Chen et al., 2019b). In this project, the effects of three factors of wormhole (A-Distance, B-Diameter, C-Content) on the imaging accuracy were studied through a mix-level factorial design with three replicates. The experimental factors and their variable levels are presented in Table 1. The factorial experimental settlement (Table 1) was produced based on the DesignExpert v10 software (Stat-Ease Inc., Minneapolis, USA). Simulated wormholes were buried at certain coordinates with var- 
iable factors. Every run was duplicated. The coordinate of simulated wormholes was detected by ground-penetrating radar SIR 3000. The absolute difference value between the experimental and actual value were considered as the experimental responses. A smaller absolute difference value indicates a better imaging accuracy.

Table 1. $2^{3}$ Factorial Design Table

\begin{tabular}{llll}
\hline Run & $\begin{array}{l}\text { A: Distance } \\
(\mathrm{cm})\end{array}$ & $\begin{array}{l}\text { B: Diameter } \\
(\mathrm{cm})\end{array}$ & $\begin{array}{l}\text { C: Dielectric } \\
\text { constant }\end{array}$ \\
\hline 1 & 13 & 1.7 & Air \\
2 & 13 & 3 & Air \\
3 & 29 & 1.7 & Air \\
4 & 29 & 3 & Air \\
5 & 13 & 1.7 & Water \\
6 & 13 & 3 & Water \\
7 & 29 & 1.7 & Water \\
8 & 29 & 3 & Water \\
9 & 13 & 1.7 & Oil and water \\
10 & 13 & 3 & Oil and water \\
11 & 29 & 1.7 & Oil and water \\
12 & 29 & 3 & Oil and water \\
\hline
\end{tabular}

Factorial effects in a mix-level factorial design can be estimated using a multiple regression model (Jiang et al., 2017; Song et al., 2018; Liu et al., 2020; Zhao et al., 2020). The regression model for the experimental design that contains 12 groups of observed data can be expressed as:

$Y=b_{0}+b_{1} X_{1}+b_{2} X_{2}+b_{3} X_{3}+b_{4} X_{1} X_{2}+$

$b_{5} X_{1} X_{3}+b_{6} X_{2} X_{3}+b_{7} X_{1} X_{2} X_{3}$

where $Y$ is the global mean; $X_{1}, X_{2}, X_{3}$ are independent variables; $b_{i}$ is the coefficient for the main and interactive effects of the factors.

\section{Results Analysis}

\subsection{Calibration of the Dielectric Constant}

In this section, the detailed process for the calibration of dielectric constant is presented. As discussed in previous sections, the radar measures the time between the emission and the reception of the signal after it is reflected by the object, as shown in Equation (2):

$2 d=v t$

where $d$ is the distance between the surface and the object, $v$ is the speed of light in the medium, and $t$ is the time measured by radar. In order to calculate the distance of the object, the speed of light in the medium, $v$, is needed. It is given in Equation (3):

$$
v=c / \sqrt{\mu_{r} \varepsilon_{r}}
$$

where $c$ is the speed of light in vacuum, $\mu_{r}$ is the relative per- meability, and $\varepsilon_{r}$ is the relative permittivity or dielectric constant.

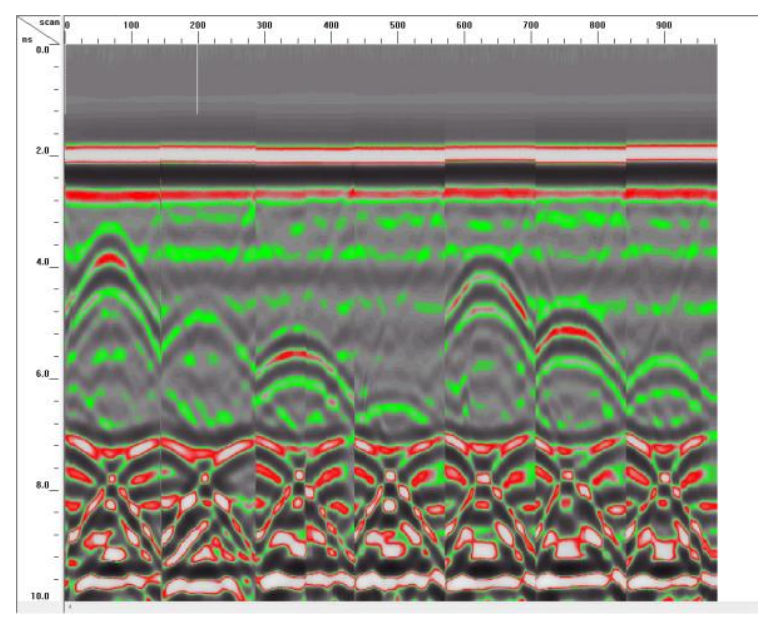

Figure 2. Radar measurements for the calibration of the dielectric constant.

Table 2. Distance and Time Measurements for the Calibration of the Dielectric Constant

\begin{tabular}{lll}
\hline Measurement number & Distance $(\mathrm{cm})$ & Time $(\mathrm{ns})$ \\
\hline 1 & 12.0 & 1.50 \\
2 & 20.0 & 2.50 \\
3 & 26.5 & 3.25 \\
4 & 35.5 & 1.25 \\
5 & 15.0 & 1.75 \\
6 & 23.0 & 2.80 \\
7 & 28.5 & 3.50 \\
\hline
\end{tabular}

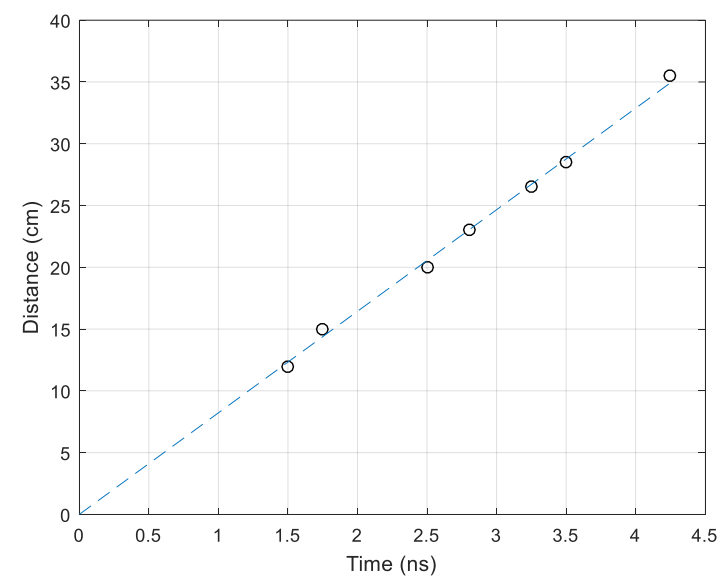

Figure 3. Linear regression for the calibration of the dielectric constant.

For this experiment, the medium is dry sand; therefore, $\mu_{r}$ is assumed to be 1 . The dielectric constant of dry sand, however, varies between $3 \sim 6$. An exact dielectric constant is needed in order to calculate distance from radar time measurements. Therefore, proper calibration is needed. The cali- 


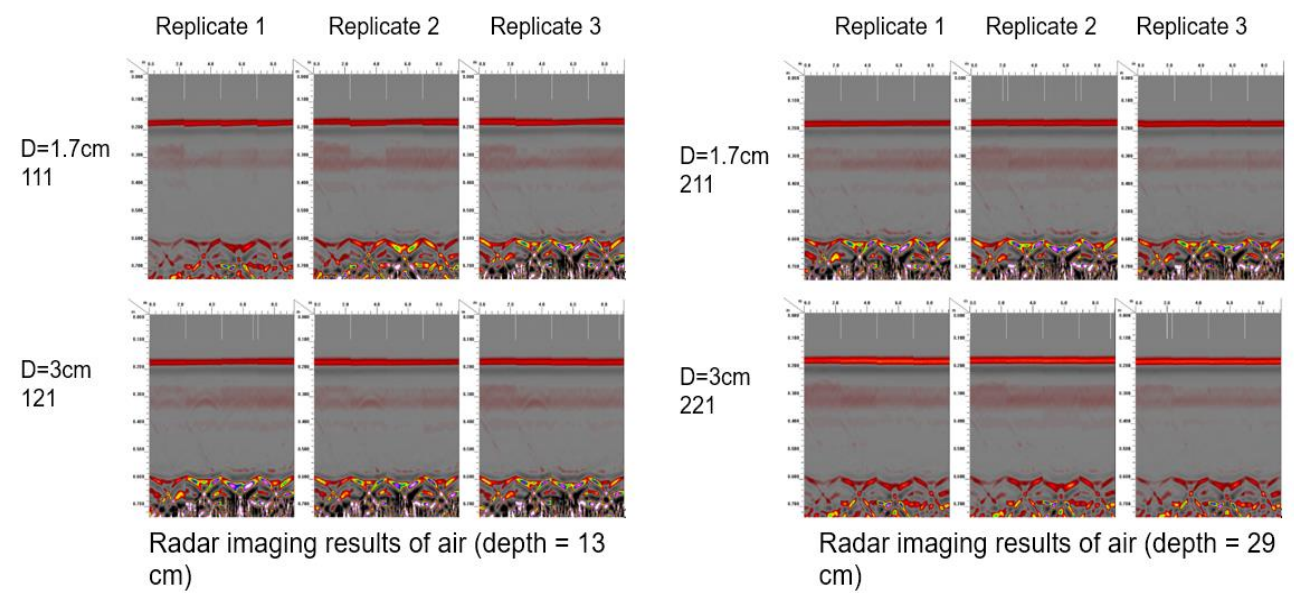

Figure 4. Comparison results of void wormhole of different diameters (Depths of the container in the left and right panel are respectively 13 and $29 \mathrm{~cm}$; the first and second row are for Diameters 1 and 2, respectively).

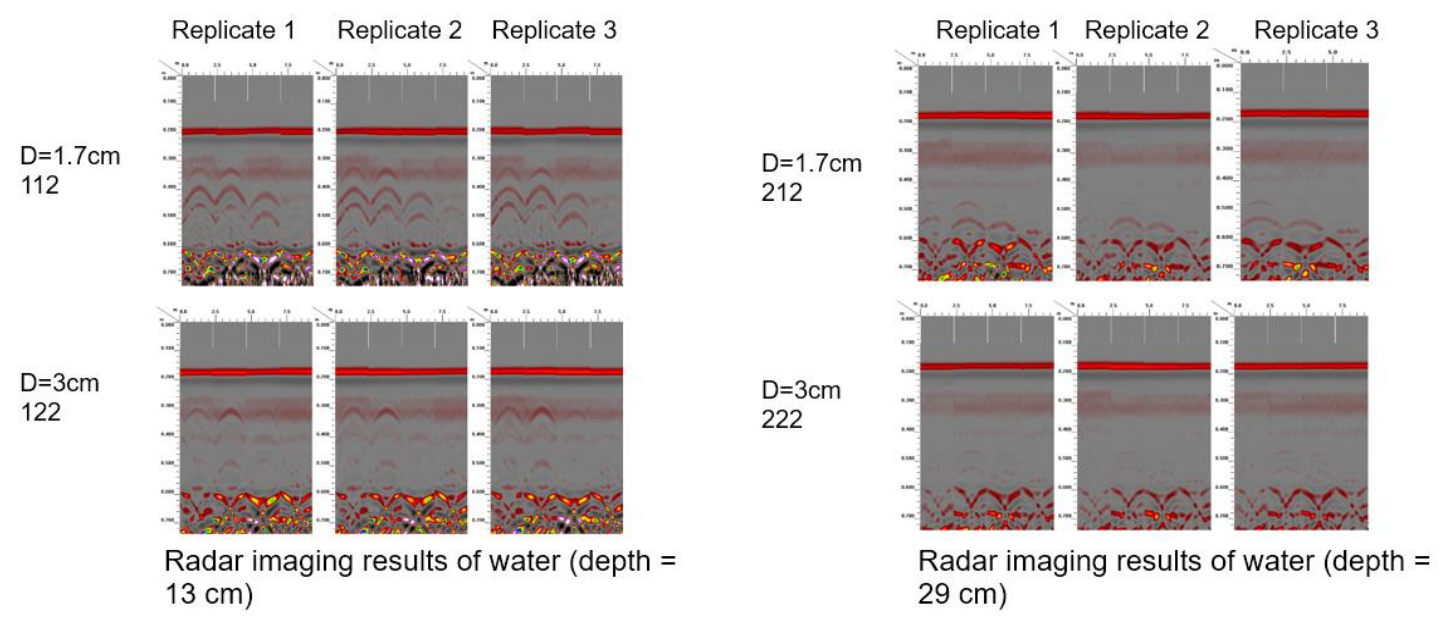

Figure 5. Comparison results of water filled wormhole of different diameters (Depths of the container in the left and right panel are respectively 13 and $29 \mathrm{~cm}$; the first and second row are for Diameters 1 and 2, respectively).

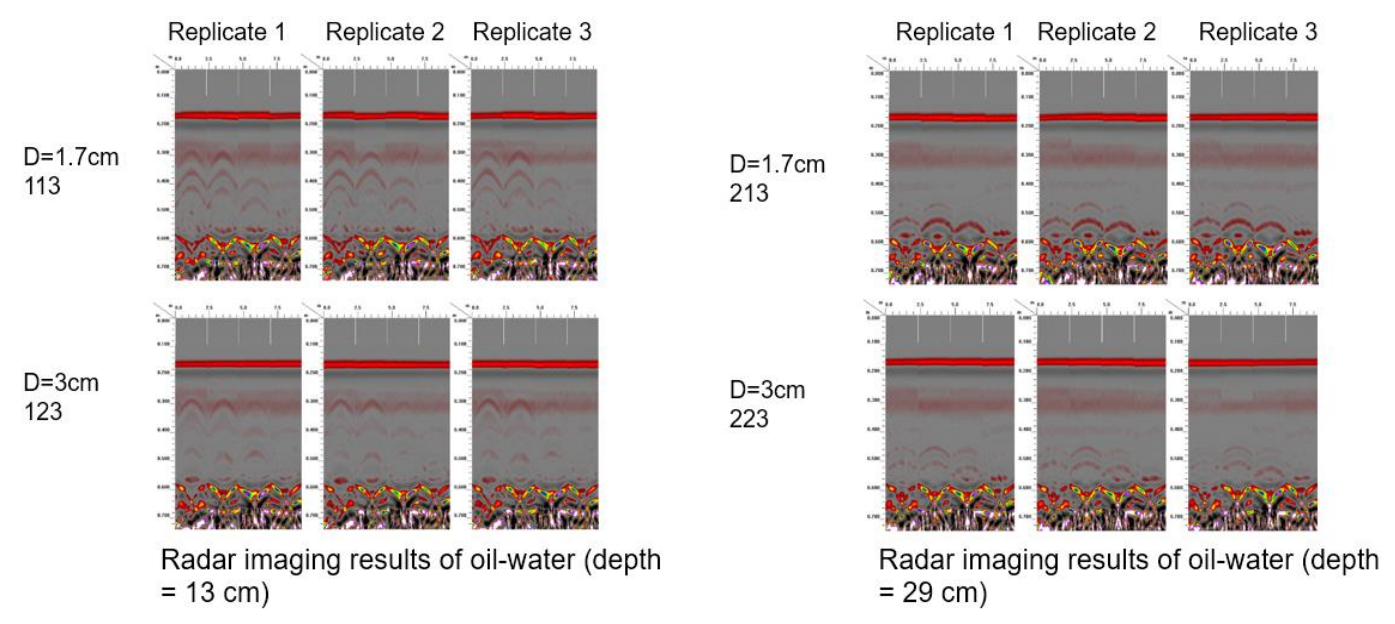

Figure 6. Comparison results of oil/water filled wormhole of different diameters (Depths of the container in the left and right panel are respectively 13 and $29 \mathrm{~cm}$; the first and second row are Diameters 1 and 2, respectively). 
bration is accomplished through multiple measurements of distance and time followed by linear regression. Seven groups of measurements were conducted. For each group of measurement, a metal bar was placed in the sand and the distance between the metal bar and the sand surface was measured. Then, radar was used to swipe the sand and a radar gram with the characteristic parabola shape was obtained. The peak of the parabola indicated that the radar was right on the top of the metal bar; therefore, the time recorded at this location was the time it took for the signal to travel from the antenna to the metal bar and back to the antenna. The 7 radar grams are shown in Figure 2. The distance and time are listed in Table 2.

For the linear regression, a relationship between distance and time is needed. It can be obtained by substituting Equation (3) into Equation (2). The result is given in Equation (4). Then, a linear regression model can be constructed using the least square method, with the constant term forced to be zero (i.e., using $d=k \times t$ as the model). Figure 3 shows the 7 groups of measurements as well as the linear regression model. The slope of the line can be used to calculate the dielectric constant, which is shown in Equation (5). The calibrated dielectric constant of the medium is 3.33 :

$$
\begin{aligned}
& d=\frac{c}{2 \sqrt{\varepsilon_{r}}} t \\
& \varepsilon_{r}=\left(\frac{c t}{2 d}\right)^{2}=\left(\frac{c}{2 \times \text { slope }}\right)^{2}
\end{aligned}
$$

\subsection{Results for Different Contents}

The study consists of scanning different objects that are buried within the sand and analyzing the effects on the scanning results in terms of different pre-assigned parameters. The blank and metal test is used to serve as a baseline test to ensure the accuracy of the scanning process. The blank and metal experiment serves as a base analysis in order to test the radar's capability in detecting any objects within the sand. The metal is put into the sand at two depths (i.e., 0.13 and $0.29 \mathrm{~m}$ ) and the results of the scanning can be found in Table 3. It can be concluded that the signal for the metal is very strong no matter which distances the metal is placed into the sand.

Table 3. Results for the Blank Experiment and Experiment on Metal

\begin{tabular}{llll}
\hline & $\begin{array}{l}\text { Actual depth of } \\
\text { container }(\mathrm{m})\end{array}$ & $\begin{array}{l}\text { Depth from } \\
\text { replicate 1 }(\mathrm{m})\end{array}$ & $\begin{array}{l}\text { Depth from } \\
\text { replicate 2 }(\mathrm{m})\end{array}$ \\
\hline Blank 0 & N/A & N/A & N/A \\
Metal 1 & 0.130 & 0.140 & 0.125 \\
Metal 2 & 0.290 & 0.280 & 0.275 \\
\hline
\end{tabular}

For the actual experiment, each of the containers will be filled with either air, water, or a mixture of oil and water. The containers are of different diameters (i.e., 1.7 and $3.0 \mathrm{~cm}$ ), which are then being put into the sand one at a time at different depths (i.e., 13 and $29 \mathrm{~cm}$ ). Radar is used to scan the containers along the horizontal surface of the sand in order to see if the containers can be observed and to identify the impacts of different depths and diameters (of the container filled with oil and water) on the results from the radar detection. For example, the container filled with water is scanned four times according to the four possible combinations of parameter settings, and 3 replicates were performed for each of the groups. The results can be found in Table 4 .

In conclusion, all the results showed that when the radar was moving along the surface of the sand, the observed signal indicating the position of the container (placed inside the sand) varies. Moreover, for different groups of the experiment, the observed signals also vary since the depths and diameters of the container are different. As a result, the distance and size of the container have some influences on the results from the scanning, and it can also be concluded that minor biases among three replicates are actually coming from the people who take the measurement and read the number on the axis.

The results indicated that the scanned signal would be significantly different between two wormhole depths from the top surface. It is indicated that the scanned signal from the smaller depth wormhole with air is much stronger than that is farther to the top surface. The results also showed that a stronger signal could be obtained from a smaller depth wormhole with water. As shown in Figures $4 \sim 6$, a smaller depth wormhole with oil-water could have much stronger signals.

Moreover, the results shown in Figures $4 \sim 6$ also indicated that the scanned signal would be significantly different between the two wormhole diameters. It is showed that the scanned signal for wormhole with a larger diameter is much stronger than that with a smaller diameter. The signals for $1.7 \mathrm{~cm}$ air are almost invisible, indicating the limitation in terms of diameter is between $1.7 \sim 3 \mathrm{~cm}$. For wormhole filled with water, the signal is stronger. A smaller wormhole has a stronger signal than that of the larger diameter. For wormhole filled with oilwater, the signal is weaker than water. Still, a smaller wormhole has a stronger signal than that of larger diameters.

\subsection{Factorial Analysis}

Analysis of variance (ANOVA) was presented to calculate the significance level of the individual and interactive effects. The ANOVA assumptions, including the normality and homogeneity of residuals, were verified in Figure 7 and Figure 8, respectively. Eight groups were selected from the above 12 ones, in order to conduct a full 23 factorial analysis with combinations of different parameters, including depth (13 and 29 $\mathrm{cm}$ ), diameter $(1.7$ and $3 \mathrm{~cm}$ ), and contents (air and water). The dielectric constant of air and water is 1 and 79, respectively. This design ensured that the primary and interactive effects were apparent.

Table 5 presented the ANOVA result for the selected 23 factorial design. The significance level of 0.05 is used in this study. It is implied that the model is significant, as seen from its F-value of 2323.15 in the table. Moreover, A, B, C, AB, AC, $\mathrm{BC}, \mathrm{ABC}$ are significant model terms since their values are 
Table 4. Results for the Containers with Air, Water, or a Mixture of Oil and Water

\begin{tabular}{lllllll}
\hline & Replicate 1 & \multicolumn{3}{l}{ Replicate 2 } & \multicolumn{2}{l}{ Replicate 3} \\
\hline & $1.7 \mathrm{~cm}$ & $3.0 \mathrm{~cm}$ & $1.7 \mathrm{~cm}$ & $3.0 \mathrm{~cm}$ & $1.7 \mathrm{~cm}$ & $3.0 \mathrm{~cm}$ \\
\hline Depth 1 $(13 \mathrm{~cm}):$ & & & & & & \\
Air & N/A & 0.14 & N/A & 0.14 & N/A & 0.145 \\
Water & 0.13 & 0.125 & 0.125 & 0.13 & 0.13 & 0.12 \\
Oil and water & 0.135 & 0.12 & 0.135 & 0.13 & 0.125 & 0.125 \\
\hline Depth 2 $(29 \mathrm{~cm}):$ & & & & & \\
Air & N/A & 0.3 & N/A & 0.35 & N/A & 0.295 \\
Water & 0.29 & 0.285 & 0.288 & 0.29 & 0.3 & 0.29 \\
Oil and water & 0.3 & 0.285 & 0.295 & 0.29 & 0.295 & 0.29 \\
\hline
\end{tabular}

Table 5. ANOVA for Factorial Model

\begin{tabular}{llllll}
\hline Source & Sum of Squares & df & Mean Square & F Value & $p$-value Prob $>$ F \\
\hline Model & 2561.28 & 7 & 365.89 & 2323.16 & $7.72 \mathrm{E}-23$ \\
A-Distance $(\mathrm{cm})$ & 64.68 & 1 & 64.68 & 410.68 & $7.81 \mathrm{E}-13$ \\
B-Diameter $(\mathrm{cm})$ & 43.20 & 1 & 43.20 & 274.29 & $1.71 \mathrm{E}-11$ \\
C-Dielectric constant $(\mathrm{cm})$ & 2227.23 & 1 & 2227.23 & 14141.12 & $5.23 \mathrm{E}-25$ \\
AB & 39.02 & 1 & 39.02 & 247.71 & $3.71 \mathrm{E}-11$ \\
AC & 96 & 1 & 96 & 609.52 & $3.64 \mathrm{E}-14$ \\
BC & 39.53 & 1 & 39.53 & 250.96 & $3.36 \mathrm{E}-11$ \\
ABC & 51.63 & 1 & 51.63 & 327.79 & $4.41 \mathrm{E}-12$ \\
Pure Error & 2.52 & 16 & 0.16 & & Significant \\
Cor Total & 2563.79 & 23 & & & \\
R-Squared & 0.999017083 & & & & \\
Adeq Precision & 125.5480262 & & & & \\
\hline
\end{tabular}

smaller than 0.05 . Figure 9 provided significant levels of primary and interactive effects through the Pareto chart. Moreover, Table 6 and Figure 10 depict the significant effects and contributions to different values. As for the main effect plot (Figure 10), the vertical distance of each line implies the main effect, while a solid line linking the mean values at different levels indicates the factor (Jiang et al., 2017).

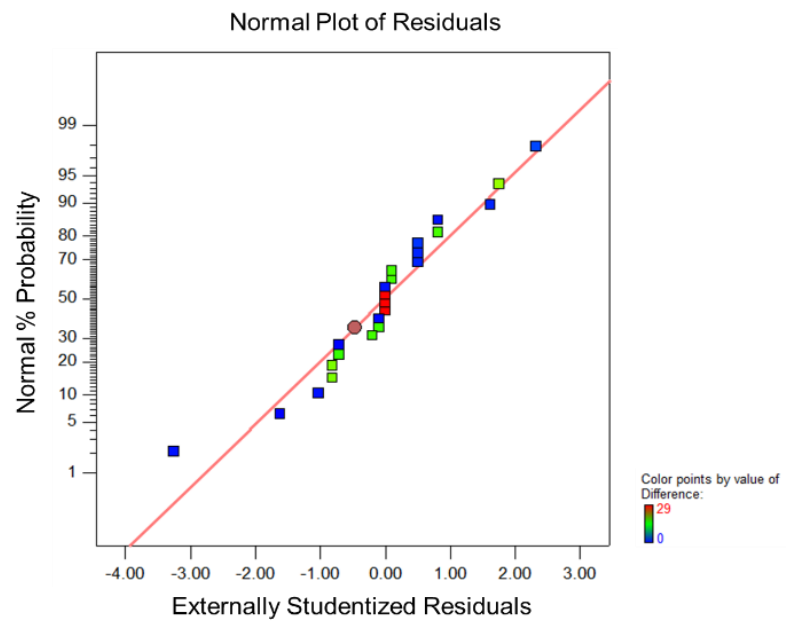

Figure 7. The normal plot of residuals.

As shown in Table 6 and Figure 10, factor C-Dielectric constant had the most significant negative effect on the difference values. The increase of dielectric constant contributed $86.87 \%$ to the decrease of difference value, significantly improving the imaging accuracy. A higher dielectric constant may lead to electromagnetic wave weakening, which improved the precision of electromagnetic wave reception. This is coincident with the results of Figure 4 to Figure 6, which show the mappings of the wormhole position. Factor B-Diameter presented a moderate effect on improving imaging accuracy. An increase in wormhole diameter leads to a decrease in difference value to some extent. Comparatively, the increase of factor ADistance had some positive effects on difference value, decreasing the imaging accuracy.

Table 4. Factor Effects and Contributions

\begin{tabular}{lll}
\hline Term & Standardized effect & Contribution $(\%)$ \\
\hline A-Distance & 3.28 & 2.52 \\
B-Diameter & -2.68 & 1.68 \\
C-Dielectric constant & -19.27 & 86.87 \\
AB & -2.55 & 1.52 \\
AC & -4.00 & 3.74 \\
BC & 2.57 & 1.54 \\
ABC & 2.93 & 2.01 \\
\hline
\end{tabular}

All the 2-factor interaction plots and the cube plot for $\mathrm{CDH}$ are shown in Figure 11. It was noted that all 2-factor interaction effects were significant. Based on Figure 11(a), it can be seen 
that the effect of diameter on imaging accuracy was not very significant at the low level of distance. However, the difference value under the high level of distance obviously decreased with the increase of diameter. The increase in wormhole distance means the increased difficulty of electromagnetic wave reception to generate accurate imaging. According to Figures 11(b) to (c), there was no significant difference among different values under dielectric constant at its high level. The difference values were similar under different levels of distance and diameter. At the low level of dielectric constant, the increase of distance significantly increases the difference values, whereas the increase of diameter decreases the difference values.

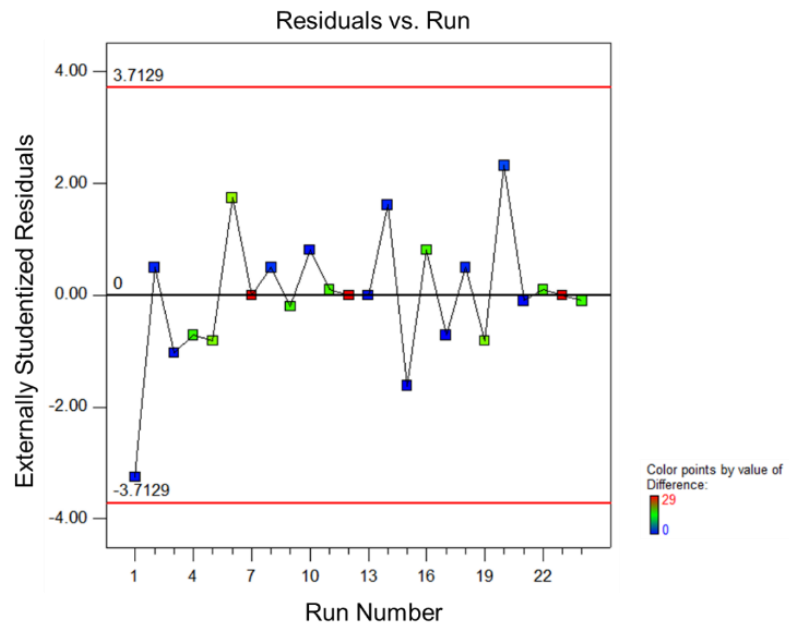

Figure 8. The plot of residuals against run numbers.

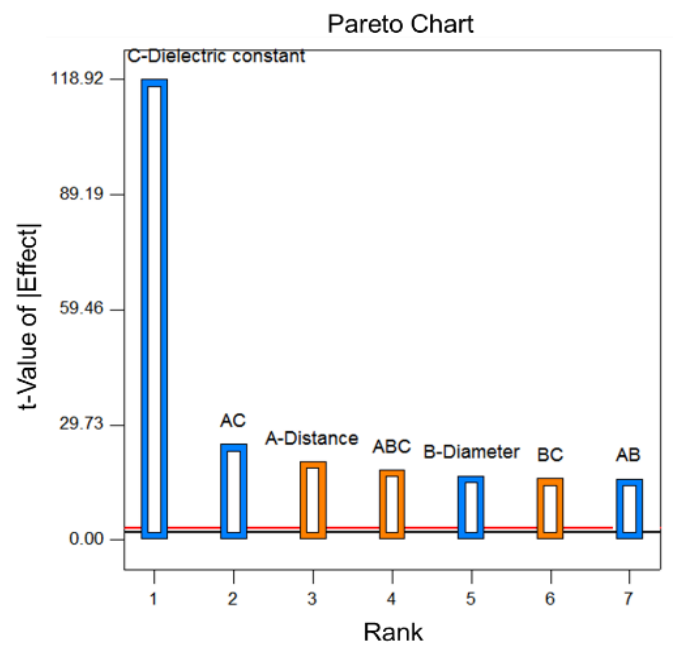

Figure 9. Pareto chart.

The cube plot for ABC (Figure 11(d)) was used to illustrate factor interactions in an intuitive manner. The wormhole diameter and distance respectively had significant negative and positive effects on different values when the dielectric constant was at its low level. The lowest difference value $(16.23 \mathrm{~cm})$ could be obtained with the wormhole diameter of $1.7 \mathrm{~cm}$ and the distance of $13 \mathrm{~cm}$. In contrast, there was no significant difference in terms of the extents of these two effects once the dielectric constant was at a high level. The changes in different values were comparatively indistinguishable, limited within 2 $\mathrm{cm}$. The scenario with the wormhole diameter of $3 \mathrm{~cm}$ and the distance of $20 \mathrm{~cm}$ could accomplish the second-lowest differrence value $(0.5 \mathrm{~cm})$, while the lowest difference value $(0.23$ $\mathrm{cm}$ ) could be found with the wormhole diameter of $1.7 \mathrm{~cm}$ and the distance of $20 \mathrm{~cm}$. Therefore, the dielectric constant can be increased to improve the imaging accuracy for further investigation according to the factorial analysis.

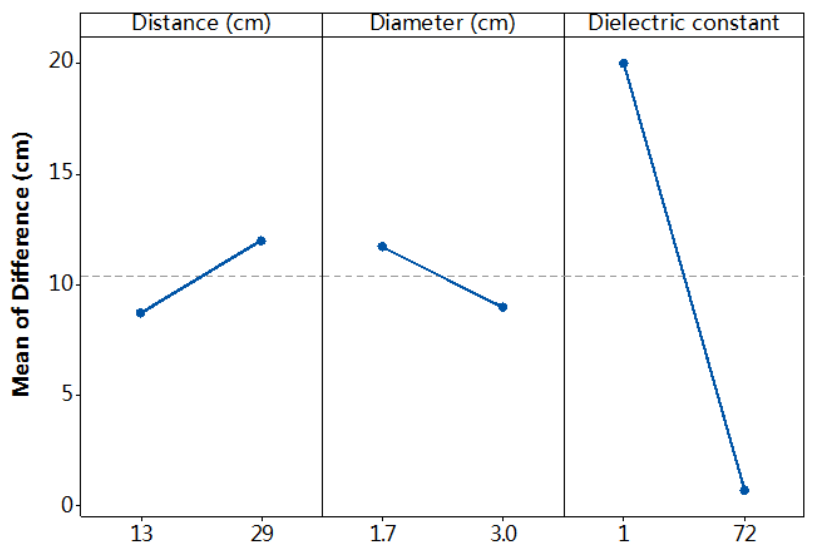

Figure 10. Main effect plot of individual parameters.

The least-squares method is employed to calculate the regression coefficients for individual and combined effects of factors. With these coefficients, regression models relative to factorial effects can be established. The final equation in terms of coded factors can be formulated as follows:

Difference $=10.35833333$

$+1.641666667 \times A-1.341666667 \times B$

$-9.633333333 \times C-1.275 \times A B-2 \times A C$

$+1.283333333 \times B C+1.466666667 \times A B C$

The developed equation can be applied to predict the response by giving levels of each factor. It is therefore helpful in identifying factor impacts through comparison of their coefficients in the equation. In particular, the high and low levels for each factor are coded as +1 and -1 , respectively. The final equation in terms of actual factors can be expressed as follows:

Difference $=-6.234714698$

$+1.719944023 \times$ Distance

$+7.144885338 \times$ Diameter

$+0.137919827 \times$ Dielectric Constant

$-0.535188696 \times$ Distance $\times$ Diameter

$-0.025713254 \times$ Distance $\times$ Dielectric Constant

$-0.111231492 \times$ Diameter $\times$ Dielectric Constant

$+0.007945107 \times$ Distance $\times$ Diameter $\times$ Dielectric Constant 

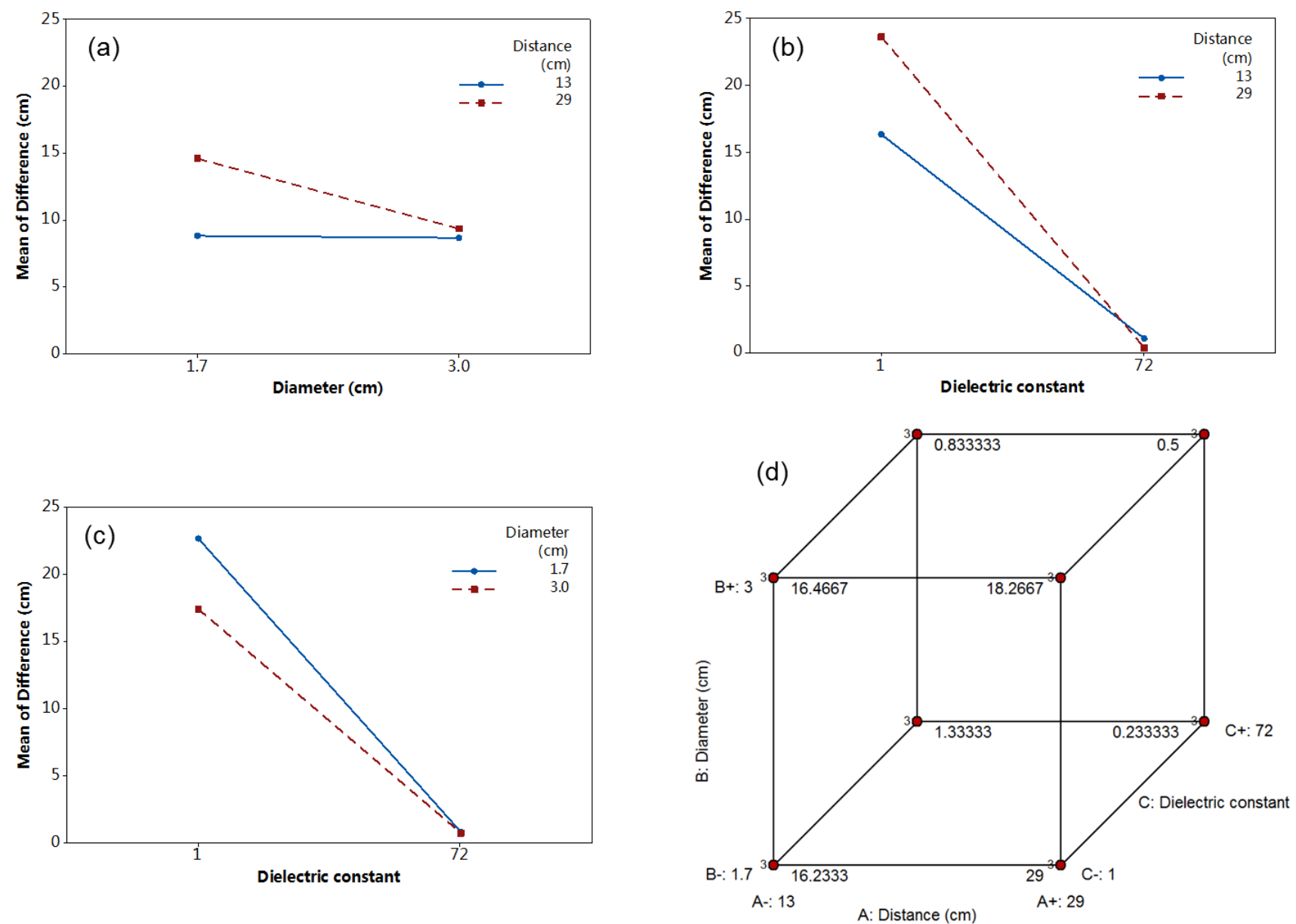

Figure 11. The 2-factor interaction plots: (a) A-Distance * B-Diameter, (b) A-Distance * C-Dielectric constant, (c) B-Diameter * C-Dielectric constant, and (d) the cube plot of ABC.

\section{Conclusion}

In this study, an integrated near-field GPR system (INFGPRs) with a frequency tunable patch antenna was developed to investigate wormholes that locate near the inspection wells. Wormholes were detected through the step-scanning against the wall of inspection wells. The inspection wells are expected to be without any metal sleeve to improve signal transmission. Herein, feasibility and availability of the developed GPR system were identified through bench-scale simulation in the lab. In addition, a factorial factor experiment was designed in order to reveal the interaction effects of impact factors (depth, size, and filler of wormhole) on the performance of the developed GPR system. An innovative feature extraction algorithm was developed for processing signals obtained from the GPR. This algorithm can fully utilize the received signal of the GPR data in different frequency bands. A generalized image processing algorithm was developed to interpret the backscatter data from enhanced signal processing. These data were stored as the raster or matrix values to their corresponding spatial and temporal coordinates within a dedicated computer.
Acknowledgments. This research was supported by the Petroleum Technology Research Centre, the National Key Research and Development Plan (2016YFC0502800, 2016YFA0601502), the Natural Sciences Foundation (51520105013, 51679087), the 111 Program (B14008), the Canada Foundation for Innovation (CFI), the Canada Research Chairs Program (CRC), and the Natural Science and Engineering Research Council of Canada (NSERC).

\section{References}

Akhter, A. and Azam, S. (2019). Flood-drought hazard assessment for a flat clayey deposit in the Canadian prairies, Journal of Environmental Informatics Letters, 1(1), 8-19. https://doi.org/1.10. 3808/jeil.201900002

John, B., Frank, D., and John, B. (2017). Experimental evaluation of several key factors affecting root biomass estimation by $1500 \mathrm{mhz}$ ground-penetrating radar, Remote Sensing, 9(12), 1337. https:// doi.org/10.3390/rs9121337

Bell, T., Daly, J., Kelley, A., and Renouf, M. A. P. (2005a). Application of ground-penetrating radar to mapping archaeological features at the Gould site, Port au Choix. Newfoundland and Labrador Studies 20(1).

Benedetto, A. and Paiewski, L. (2015). Civil Engineering Applications of Ground Penetrating Radar. https://doi.org/10.1007/978-3-31904813-0 
Chen, X.J., Huang, G.H., An, C.J., Feng, R.F., Wu. Y.H., and Huang, C. (2019a). Plasma-induced PAA-ZnO coated PVDF membrane for oily wastewater treatment: Preparation, optimization, and characterrization through Taguchi OA design and synchrotron-based X-ray analysis, Journal of Membrane Science, 582, 70-82. https://doi. org/10.1016/j.memsci.2019.03.091

Chen, X.J., Huang, G.H., An, C.J., Feng, R.F., Yao, Y., Zhao, S., Huang, C., and Wu. Y.H. (2019b). Plasma-induced poly(acrylic acid)- $\mathrm{TiO}_{2}$ coated polyvinylidene fluoride membrane for produced water treatment: Synchrotron X-Ray, optimization, and insight studies, Journal of Cleaner Production, 227, 772-783. https://doi.org/10. 1016/j.jclepro. 2019.04.226

Duisterwinkel, E.H.A., Dubbelman, G., Demi, L., Talnishnikh, E., Bergmans, J.W.M., and Wörtche, H.J. (2016). Mapping swarms of resource-limited sensor motes: solely using distance measurements and non-unique identifiers, 2016 IEEE Symposium Series on Computational Intelligence (SSCI), IEEE, 2016.

Gentili, G.G. and Spagnolini, U. (2000). Electromagnetic inversion in monostatic ground penetrating radar: TEM horn calibration and application, IEEE Transactions on Geoscience and Remote Sensing, 38(4), 1936-1946. https://doi.org/10.1109/36.851775

Istchenko, C.M. and Gates, I.D. (2014). Well/wormhole model of cold heavy-oil production with sand, SPE Journal, 19(02), 260-269. https://doi.org/10.2118/150633-PA

Jiang, Y., Zhang, Y., Banks, C., Heaven, S., and Longhurst, P. (2017). Investigation of the impact of trace elements on anaerobic volatile fatty acid degradation using a fractional factorial experimental design, Water Research, 125, 458-465. https://doi.org/10.1016/j. watres.2017.09.010

Khristoforov, I. and Omelyanenko, A. (2018). Improving the efficiency of hydrological investigations by submersible ground Penetrating Radar, IEEE Geoscience and Remote Sensing Letters, 15(3), 335-339. https://doi.org/10.1109/LGRS.2017.2786858

Liu, X. and Zhao, G. (2004). Effects of wormholes oncold heavy oil production, Canadian International Petroleum Conference, Petroleum Society of Canada, Calgary, Alberta, 2004. https://doi.org /10.2118/2004-048

Liu, X. and Zhao, G. (2005). Transient pressure behavior of cold heavy oil production wells, SPE International Thermal Operations and Heavy Oil Symposium. Society of Petroleum Engineers, Calgary, Alberta, Canada, 2005.

Liu, Y.Q., Huang, G.H., An, C.J., Chen, X.J., Zhang, P., Feng, R.F., and Xiong, W.H. (2020). Use of Nano-TiO2 self-assembled flax fiber as a new initiative for immiscible oil/water separation, Journal of Cleaner Production, 249, 119352. https://doi.org/10.1016/j. jclepro.2019.119352

Natural Resources Canada (2019). Crude oil facts. https://www.nrcan. gc.ca/science-data/data-analysis/energy-data-analysis/energy-facts/ crude-oil-facts/20064.

Pajewski, L., Benedetto, A., Derobert, X., Giannopoulos, A., Loizos, A., Manacorda, G., Marciniak, M., Plati, C., Schettini, G., and Trinks, I. (2013). Applications of ground penetrating radar in civil engineering-COST action TU1208, 2013 7th International Workshop on Advanced Ground Penetrating Radar, IEEE, 2013. https://doi.org/10.1109/IWAGPR.2013.6601528

Rangriz Shokri, A. and Babadagli, T. (2014). Modelling of cold heavyoil production with sand for subsequent thermal/solvent injection applications, Journal of Canadian Petroleum Technology, 2014, 53(02), 095-108. https://doi.org/10.2118/158934-PA

Saarenketo, T. and Scullion, T. (2000). Road evaluation with ground penetrating radar, Journal of Applied Geophysics, 43(2), 119-138. https://doi.org/10.1016/S0926-9851(99)00052-X.

Sanyal, T. and Al-Sammak, I. (2011). Analysis of the first CHOPS pilot for heavy oil production in Kuwait, Canadian Unconventional Resources Conference, Society of Petroleum Engineers, Calgary, Alberta, Canada, 2011.

Sawatzky, R.P., Lillico, D.A., London, M.J., Tremblay, B.R., and Coates, R.M. (2002). Tracking cold production footprints, Canadian International Petroleum Conference, Petroleum Society of Canada Calgary, Alberta, Canada, 2002.

SHRP2 (2013). Mapping Voids, Debonding, Delaminations, Moisture, and Other Defects Behind or Within Tunnel Linings, The National Academies Press, Washington, DC.

Song, P., Huang, G.H., An, C.J., Shen, J., Zhang, P., Chen, X.J., Shen, J., Yao, Y., Zheng, R.N., and Sun, C.X. (2018). Treatment of rural domestic wastewater using multi-soil-layering systems: Performance evaluation, factorial analysis and numerical modeling, Science of The Total Environment, 644, 536-546. https://doi.org/10.1016/j. scitotenv.2018.06.331.

Zhou, Y., Huang, G., Zhu, H., Li, Z., and Chen, J. (2016). A factorial dual-objective rural environmental management model, Journal of Cleaner Production, 124, 204-216. https://doi.org/10.1016/j.jcle pro.2016.02.081.

Zhao, Y., Huang, G., An, C., Huang, J., Xin, X., Chen, X., Hong, Y. and Song, P. (2020). Removal of Escherichia Coli from water using functionalized porous ceramic disk filter coated with $\mathrm{Fe} / \mathrm{TiO}_{2}$ nanocomposites. Journal of Water Process Engineering, 33, p.101013. https://doi.org/10.1016/j.jwpe.2019.101013 